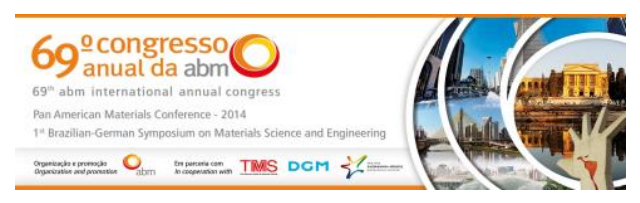

Tema: Gestão sistêmica

\title{
INFORMATIZAÇÃO DA GESTÃO DA PRODUÇÃO EM UMA FORJARIA NO SETOR AUTOMOTIVO*
}

\section{Resumo}

Alexandre Rodizio Bento ${ }^{1}$

Élcio Miguel Prus ${ }^{2}$

Eduardo Fagundes Falconelli ${ }^{3}$

A utilização da tecnologia na gestão dos processos de produção em forjaria, tornase cada vez mais imprescindível para se manter competitivo no setor automotivo. Com isso, a necessidade de ferramentas tecnológicas se faz presente para obter controles ágeis e recursos otimizados. A informatização é uma destas ferramentas que podem ser usadas na gestão da produção no setor automotivo. Esta permite que os gestores consigam monitorar em tempo real a produção e caso necessário intervir no processo de forma ágil para eliminar ou diminuir as não conformidades durante a produção. Este trabalho discute a informatização da gestão da produção em uma forjaria no setor automotivo e a importância das ferramentas tecnológicas aplicadas como suporte a gestão. Apresenta-se um caso real aplicado no setor, onde a informatização apresentou ganhos de acesso à informação e agilidade na tomada de decisão. Neste, realiza-se uma comparação do processo de gestão manual com a proposta de informatização. Como resultado, verificou que as ferramentas tecnológicas aplicadas possibilitaram melhorar fortemente a gestão durante o processo produtivo e mostrou-se superior nos quesitos de qualidade e tempo para difundir a informação, bem como reduzir custos operacionais.

Palavras-chave: Informatização; Gestão da produção; Forjaria; Setor automotivo.

\section{COMPUTERIZATION THE PRODUCTION MANAGEMENT IN AN AUTOMOTIVE SECTOR FORGING}

\section{Abstract}

The use of technology in the management of production processes in forging becomes increasingly essential to remain competitive in the automotive sector. With this, the need for technological tools is present for agile controls and optimized resources. Computerization is one of those tools that can be used in the automotive industry production management. This allows managers to monitor real time production and if necessary intervene in the process in an agile way to eliminate or reduce non-conformities during production. This paper discusses the computerization of production management in a forge in the automotive sector and the importance of technological tools applied to support management. It presents a real case applied in the sector, where computerization showed gains access to information and agility in decision-making. In this, it holds a comparison of manual management process with the proposed computerization. As a result, discovered that technology- enabled greatly improved the management during the production process and proved to be superior in quality matters and time to disseminate information as well as reduce operating costs.

Keywords: Computerization; Production management; Forging; Automotive sector.

1 Processamento de Dados, Mestre em Desenvolvimento de Tecnologia, Faculdades Santa Cruz, Curitiba, PR, Brasil.

2 Bacharel Sistemas de Informação, Mestre em Eng.de Produção, Faculdades Santa Cruz, Curitiba, PR, Brasil.

3 Graduando em Engenharia de Produção, Faculdade FACEAR, Curitiba, PR, Brasil.

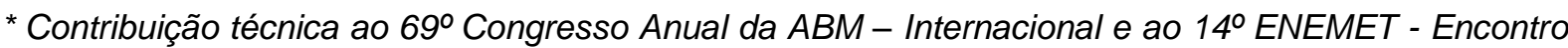
Nacional de Estudantes de Engenharia Metalúrgica, de Materiais e de Minas, 21 a 25 de julho de 2014, São Paulo, SP, Brasil. 


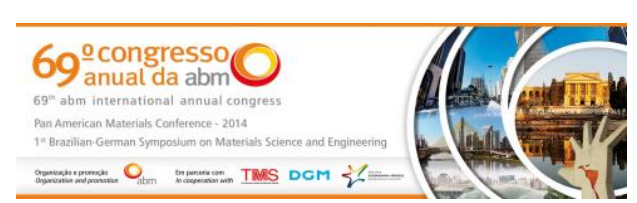

\section{INTRODUÇÃO}

A competitividade do setor automotivo, faz que as indústrias busquem cada vez mais por ferramentas tecnológicas para se manter no mercado. Uma destas ferramentas é a informatização da gestão da produção, ou seja, integrar software e hardware com máquinas automáticas no ambiente de produção [1]. Conduzir a informatização no processo de produção, torna-se um desafio, para as indústrias de forjaria, devido ao grande número de opções existentes no mercado de tecnologia e a falta de profissionais qualificados nas indústrias.

A indústria de forjaria trabalha na transformação de matéria prima bruta, o aço por exemplo em peça acabada, pela aplicação de esforços de compressão mecânica $[2,3]$. O processo de forja utiliza uma matriz superior e outra na parte inferior onde está o molde é inserida a matéria prima, quando a parte superior desce, forja a peça. Uma peça forjada é mais resistente que a fundida (utiliza liga de metal líquida para moldar a peça). Algumas máquinas utilizadas no processo de forjar tem possibilidade de integrar com sistemas de gestão para automatizar 0 processo de produção, com coleta automática dos dados em tempo real.

Para fazer a informatização dos processos de produção e obter benefícios como agilidade e recursos otimizados para a tomada de decisão, deve-se compor uma equipe com os usuários chaves do processo e das áreas envolvidas tais como: engenharia, produção, informática e outras, o apoio da alta direção é primordial neste processo [4]. Esta equipe deve elaborar um cronograma com as datas e atividades que foram levantadas com base nas necessidades da automação, o treinamento dos envolvidos é primordial. Todas as atividades a serem desenvolvidas devem ter um responsável que semanalmente deve fazer atualização do projeto.

As indústrias do setor automotivo passaram a investir mais em tecnologia moderna para maximizar os ganhos no ambiente de produção e concorrer no mercado, onde a competitividade é acirrada [5]. Máquinas de produção integradas a computadores já fazem parte dos processos produtivos das indústrias, como forma de reduzir custos operacionais com mão de obra e aumentar a produtividade em menor tempo. Além disso, a tecnologia permite obter mais assertividade nos produtos com qualidade assegurada, gerando satisfação ao cliente final.

O objetivo deste trabalho é apresentar o processo de gestão manual da produção baseado em controle de papel e planilha excel. Após esta apresentação substituir o processo manual por um informatizado com tecnologia embutida. Com isso, pretende-se demonstrar a melhor gestão da produção em tempo real, e alguns ganhos como reduzir os custos operacionais e o tempo para difundir as informações na indústria.

\subsection{Informatização da Gestão da Produção}

A informatização dos processos de gestão da produção está crescendo de forma acentuada dentro das indústrias do setor automotivo, devida a necessidade de aumentar a produtividade com menor custo [6]. Todos estes processos controlados por tecnologia vêm se tornando cada vez mais complexos e transformando em ferramentas estratégicas para tomada de decisão.

A informação que é coletada diretamente da máquina no chão de fábrica gera um impacto importante na gestão da produção, pois permite analisar e comparar os dados de produção e ocorrências de paradas por manutenção [7]. As ocorrências

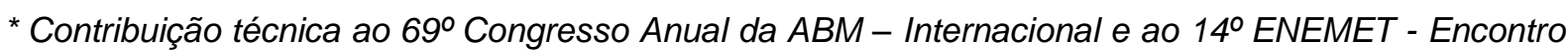
Nacional de Estudantes de Engenharia Metalúrgica, de Materiais e de Minas, 21 a 25 de julho de 2014, São Paulo, SP, Brasil.
} 


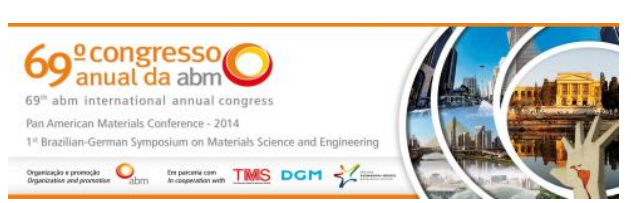

são apontadas a qualquer momento com auxílio de um sistema interligado a máquina, e gravação em banco de dados para consulta dos históricos no futuro.

As exigências de tecnologias modernas empregadas no ambiente produtivo proporcionam mais confiabilidade e flexibilidade dos recursos, além de, disponibilizar aos gestores maiores controles de forma ordenada sobre as operações de produção [8]. Ainda é possível fazer simulações de produção para atingir a melhor disponibilidade de recursos e evitar máquinas ociosas na produção.

Para o sucesso da informatização no ambiente de produção algumas etapas devem ser executadas como monitorar a funcionalidade dos equipamentos e sistemas utilizados para esta finalidade [9]. Verificar se as informações do sistema estão consistentes em relação ao físico produzido, buscar a utilização de forma efetiva das informações sistêmicas pelos gestores. Somente a partir deste momento pode-se evitar inconsistência no processo e fazer os ajustes se necessário.

Implementar tecnologias no ambiente de produção como forma de informatização contribui para alavancar os controles produtivos, pois a tecnologia muitas vezes facilita o processo e faz difundir a informação de maneira expressiva dentro da indústria [10]. Também fornece dados para a gestão da produção interativa, ou seja, pode-se interagir em tempo real no processo de fabricação e fazer ajustes, com isso obter ganhos expressivos na peça final.

As tecnologias embutidas na gestão da produção do setor automotivo permite criar indicadores de tempo de produção, paradas de máquinas, defeitos, perdas, capacidade de produção entre outros [11]. Os indicadores são fundamentais para conhecer e obter ganhos no processo produtivo, pois o planejamento da produção deve ser flexível devido a grande mudança que ocorre no mercado globalizado. Assim, atender as necessidades dos clientes devido alterações do mercado mundial, faz firmar ainda mais a parceria entre fornecedor e cliente.

Desta forma, as ferramentas tecnológicas podem revolucionar ainda mais a gestão da produção e acelerar seu manuseio [12]. Sendo estas bem aplicadas podem ser a chave para identificar peças, quantidades, baixas e registrar paradas de manutenção com agilidade. A informação correta na hora certa pode ser um diferencial estratégico para indústria, pois além difundir entre os responsáveis de forma eficaz, pode contribuir na tomada de decisão. A indústria automotiva busca cada vez mais atender as expectativas dos clientes com produtos de qualidade entregues no prazo, para isso a produção deve ser flexível. Para obter esta flexibilidade a gestão da produção em tempo real é um dos requisitos principais.

\section{MATERIAIS E MÉTODOS}

Para comprovar a eficiência da informatização na gestão da produção foi selecionada uma indústria de forjaria que atua no setor automotivo e produz bielas, cubos de roda e pontas de eixo entre outros produtos para as montadoras. A indústria tem em seu quadro funcional cento e cinquenta funcionários divididos em três turnos com vinte e dois dias por mês.

A figura 1 demonstra a gestão da produção manual, onde os custos operacionais são destacados. Além disso, apresenta o tempo gasto na gestão da produção com digitação, impressão e outros processos executados manuais.

\footnotetext{
* Contribuição técnica ao $69^{\circ}$ Congresso Anual da ABM - Internacional e ao 14ํㅡㄹ ENEMET - Encontro Nacional de Estudantes de Engenharia Metalúrgica, de Materiais e de Minas, 21 a 25 de julho de 2014, São Paulo, SP, Brasil.
} 

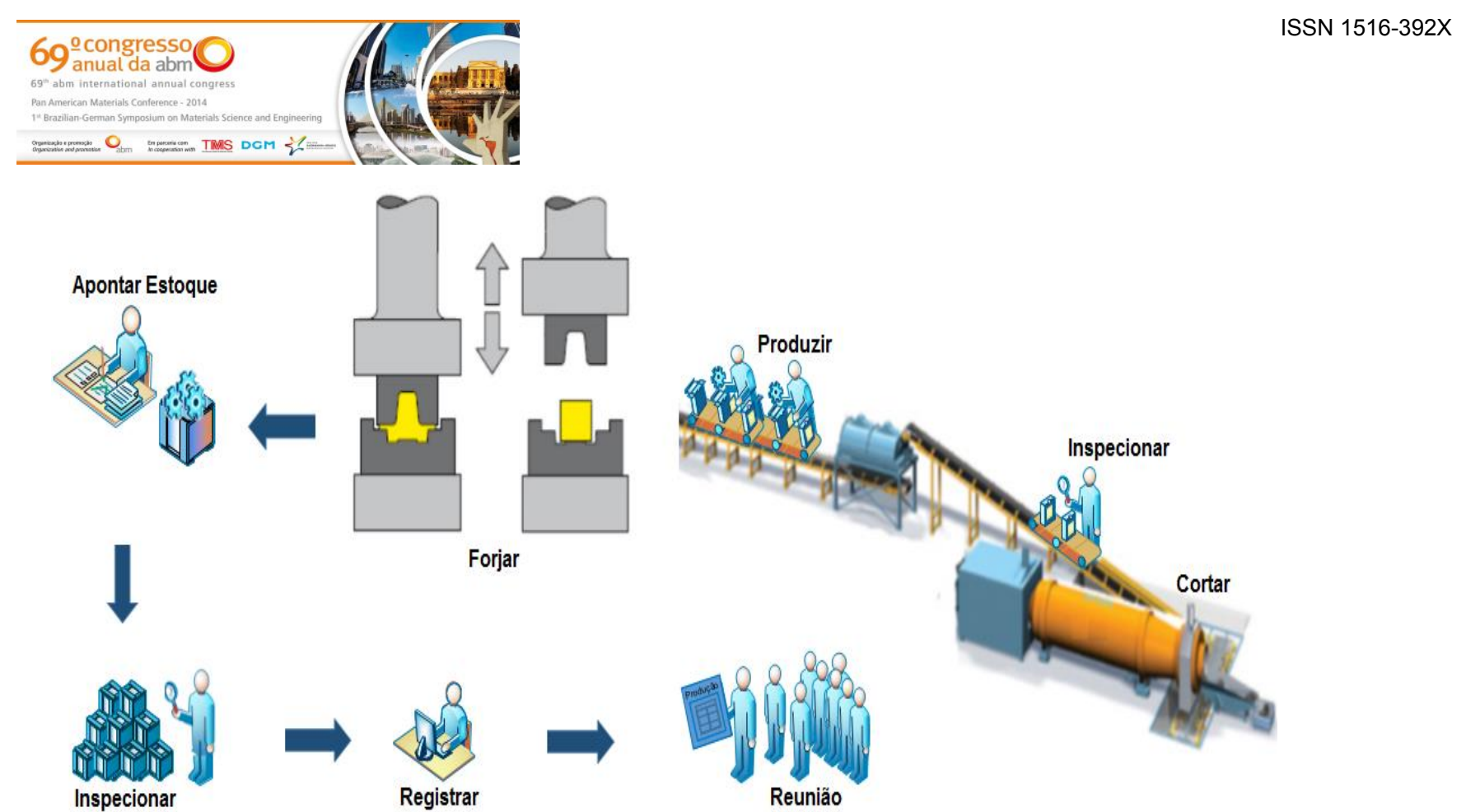

Figura 1. Fluxo do processo da gestão da produção manual.

A figura 1 demonstra o processo de gestão da produção manual para forjar bielas. 0 processo se inicia no corte do aço em pedaços, conforme o tamanho da biela a ser produzida. $\mathrm{O}$ aço cortado é chamado de tarugo e colocado em um tanque que alimenta o processo por meio de esteira. A próxima operação é aquecer o tarugo a temperatura ideal e ser analisado pelo controle de qualidade, depois disso, passar por uma preformadora (máquina com dois rolos e uma abertura no centro, onde 0 tarugo deve passar, para garantir homogeneidade no diâmetro). Em seguida 0 tarugo alimenta o molde da prensa que efetua uma batida a cada quatro segundos. Cada tarugo recebe duas batidas, a primeira modela a biela e a segunda corta rebarbas e arestas.

A cada quatro segundos são forjadas duas bielas pela prensa, que passam pela lavadora com óleo para proteger a peça contra ferrugem para se encaminhadas ao controle de qualidade, que avalia o peso e faz inspeção visual, além disso, utiliza luz ultravioleta para procurar possíveis falhas, trincas ou porosidade na peça.

O apontamento manual de produção ocorre na prensa, através do preenchimento do flip chart (tripé em madeira com folhas de papel) de hora em hora. Neste mesmo momento também são apontados os tempos e motivos dos eventos de paradas que ocorreram neste período, o tempo gasto em todos estes apontamentos são de duas horas por dia, ou seja, quarenta minutos por turno. No início de cada turno todos os apontamentos feitos em folha de papel são digitados em planilha Excel pelo analista de engenharia com tempo estimado de uma hora e trinta minutos por dia. Uma vez por semana são gerados os dados em forma quantitativa e apresentados em gráficos indicadores, para serem expostos em painéis no chão de fábrica (gestão a vista), este trabalho consome uma hora por semana ou doze minutos por dia do mesmo analista.

No período da manhã ocorre a reunião de produção no chão de fábrica onde são informadas as quantidades produzidas no dia anterior e os tempos de parada da máquina. Estas informações são impressas pelo analista de engenharia em várias cópias entregues aos responsáveis todos os dias antes de realizar a reunião, com tempo estimado de 30 minutos por dia.

Um plano de ação com as melhorias no processo de produção e nas paradas relatadas é desenvolvido com base nas informações que foram registradas, para

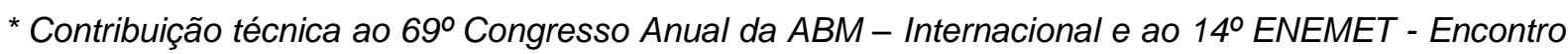
Nacional de Estudantes de Engenharia Metalúrgica, de Materiais e de Minas, 21 a 25 de julho de 2014, São Paulo, SP, Brasil.
} 


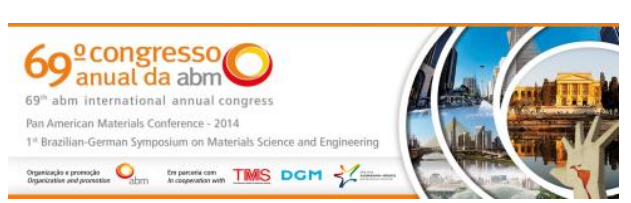

visualizadas na intranet da empresa a qualquer momento, com isso permite acesso a informação de forma ágil.

O tempo para forjar a biela continua o mesmo do processo manual, mas os ganhos com o processo de informatização são muitos, pois as quantidades de peças produzidas são analisadas e apontadas de forma automática no sistema de gestão. Pois, no dia seguinte a reunião ocorre em frente do monitor com informações em tempo real ou por consulta a históricos anteriores, com diversos dados coletados para executar ações de forma mais assertiva. Com a informatização da gestão da produção são gerados gráficos e indicadores sobre produtividade, ociosidade de máquina e tempo de paradas para manutenção entre outros, direto no monitor, sem a necessidade de impressão em papel.

\section{RESULTADOS E DISCUSSÃO}

Para uma melhor comparação entre os processos de gestão da produção manual e informatizado é necessário resgatar os fluxos destes processos. A figura 3 representa ambos os fluxos onde pode observar que a principal alteração está no processo de apontar a quantidade produzida. Na gestão informatizada este processo foi excluído por ser desnecessário, ou seja, foi substituído pela tecnologia de coletor de dados de forma automática.

A figura 3 apresenta a comparação entre os fluxos de gestão manual e informatizada, onde é possível observar diversas melhorias com utilização da tecnologia na gestão informatizada. A primeira melhoria é a substituição do processo de apontar a produção em folha de papel, pelo coletor de dados com gravação da quantidade produzida em tempo real, além de proporcionar agilidade no processo ainda armazena os dados em banco de dados com maior segurança. As informações gravadas são apresentadas em um monitor no chão de fábrica, são os diferenciais proporcionados pela integração da tecnologia de coletor de dados com o CLP da prensa.

Com a informatização o apontamento dos dados de produção ocorre de forma automática sem interferência humana, as ocorrências de paradas de manutenção no processo de produção são informadas pelo operador por uma codificação. Esta ação aliada a outras aplicada no processo de gestão informatizada possibilitou reduzir custo e tempo para digitar e imprimir os indicadores e gráficos. A tabela 1 apresenta a economia que foi proporcionada pela informatização na gestão da produção, onde o tempo em horas associada aos valores por mês foram reduzidos de forma eficaz.

Tabela 1. Economia com a gestão informatizada

\begin{tabular}{l|c|c|c|c}
\hline \multicolumn{1}{c|}{ Operações } & $\begin{array}{c}\text { Horas por } \\
\text { Dia }\end{array}$ & $\begin{array}{c}\text { Horas por } \\
\text { Mês }\end{array}$ & $\begin{array}{c}\text { Valor } \\
\text { Hora }\end{array}$ & $\begin{array}{c}\text { Valor } \\
\text { Mês }\end{array}$ \\
\hline $\begin{array}{l}\text { Apontar produção e } \\
\text { manutenção }\end{array}$ & 2 & 44 & $\mathrm{R} \$ 20,00$ & $\mathrm{R} \$ 880,00$ \\
\hline $\begin{array}{l}\text { Digitar apontamentos em } \\
\text { excell }\end{array}$ & 1,30 & 33 & $\mathrm{R} \$ 20,00$ & $\mathrm{R} \$ 660,00$ \\
\hline Gerar gestão a vista & 0,12 & 5,24 & $\mathrm{R} \$ 20,00$ & $\mathrm{R} \$ 104,80$ \\
\hline $\begin{array}{l}\text { Imprimir indicadores } \\
\text { produção e manutenção }\end{array}$ & 0,30 & 10 & $\mathrm{R} \$ 20,00$ & $\mathrm{R} \$ 200,00$ \\
\hline
\end{tabular}

A tabela 1 demonstra a redução de tempo e custos relacionados aos processos de apontar a produção e registrar as requisições na gestão manual. Os tempos gastos por dia para apontar a produção na gestão manual atinge duas horas por dia

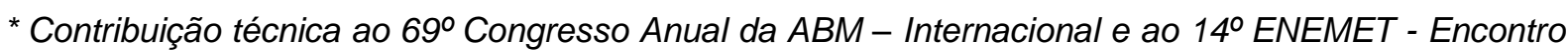
Nacional de Estudantes de Engenharia Metalúrgica, de Materiais e de Minas, 21 a 25 de julho de 2014, São Paulo, SP, Brasil. 


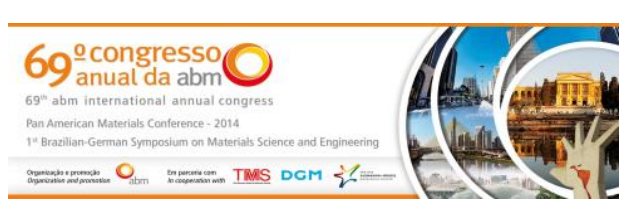

multiplicado por vinte e dois dias trabalhados resulta em quarenta e quatro horas por mês multiplicado ao custo de vinte reais a hora do engenheiro totaliza em oitocentos e oitenta reais por mês.

Digitar os apontamentos em excell para gerar índices, indicadores e gráficos gastam tempo de uma hora e meia por dia, ou seja, trinta e três horas mensais ou seiscentos e sessenta reais. Para gerar as impressões com informações para fazer a gestão a vista o tempo estimado é doze minutos por dia ou uma hora por semana ou ainda 5,24 horas por mês que totaliza cento e quatro reais e oitenta centavos mês. Para imprimir os indicadores de produção que são entregues aos gestores diariamente ocupa o tempo de trinta minutos por dia ou dez horas por mês que atinge o total de duzentos reais mês.

O total do tempo reduzido foi $(92,24)$ horas por mês, já o valor atingiu a $(R \$$ $1.844,80)$. O processo de produção informatizado ficou mais eficiente com a redução do tempo comparado ao manual. Além disso, permite vários ganhos com agilidade que a informação que chega aos gestores, um deles é a possibilidade de intervir no processo produtivo em tempo real. Com isso, aumentar a produtividade e agregar maior valor ao produto final.

As paradas de manutenção também foram reduzidas em menor tempo devido a informatização que permite agilizar o envio dos problemas encontrados seja por email, SMS ou no próprio monitor no chão de fábrica. Todos os tempos de parada de máquina foram conhecidos devido operador inserir o código da respectiva parada e muitas vezes justificar o problema. Estas ações contribuíram de forma efetiva para aumentar a disponibilidade das máquinas na produção, consequentemente pode-se produzir mais em menor tempo.

$\mathrm{Na}$ figura 3 é possível observar que a redução de tempo está relacionada a informatização da gestão da produção, que permitiu excluir dois processos de produção realizados com interferência humana, e ainda possibilitava gerar erros para apontar e registrar a quantidade produzida. A informatização gerou maior confiabilidade e segurança nas informações da produção, pois os dados são coletados de forma automática sem interferência humana que contribui para difundir as informações entre diversos departamentos da indústria e até ao cliente.

\footnotetext{
* Contribuição técnica ao $69^{\circ}$ Congresso Anual da ABM - Internacional e ao 14ํㅡㄹ ENEMET - Encontro Nacional de Estudantes de Engenharia Metalúrgica, de Materiais e de Minas, 21 a 25 de julho de 2014, São Paulo, SP, Brasil.
} 


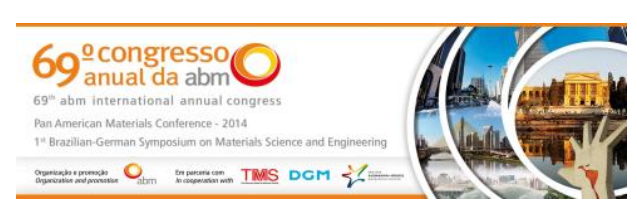

\section{CONCLUSÃO}

A complexidade observada na gestão da produção no setor automotivo, associada as tecnologias aplicada no setor, obrigam as indústrias investir cada vez mais em ferramentas modernas que permitam melhorar a gestão da produção. A informatização é uma dessas tecnologias que se bem empregada e utilizada pode elevar a capacidade operacional e a produtividade da indústria, além de ser um diferencial estratégico para tomada de decisão.

A informatização é uma ferramenta que contribui de forma eficaz para monitorar e gerar informações em tempo real sobre a produção, e assim permitir intervir nos processos produtivos em menor tempo. Além disso, pode proporcionar um comparativo de indicadores, tais como: quantidade produzida por turno em relação a meta, tempo de paradas de produção e as ações de melhorias que foram realizadas por turno.

Com base nos resultados obtidos no processo de informatização, pode-se afirmar que a escolha adequada de tecnologias compatíveis, bem como envolvimento direto da equipe responsável pela informatização, é primordial para o desempenho eficaz da gestão da produção. Contudo, faz-se necessário o comprometimento dos responsáveis pelas áreas de produção, manutenção e informática, onde as adequações e alterações devem ser atendidas de forma ágil, para evitar perdas no ambiente produtivo, e ainda ser flexível e compatível com as mudanças necessárias. Todas integrações entre tecnologias e pessoas envolvidas, fazem aumentar os ganhos e de forma orquestrada melhorar cada vez mais o processo de gestão da produção.

\section{REFERÊNCIAS}

1 Gouveia LB, Ranito J. Sistemas de Informação de Apoio à Gestão. Porto: SPI, 2004.

2 Polozine A. Desenvolvimento da Técnica Analítica para Determinar a Resistência Térmica de Contato no Processo de Forjamento. Tese (Doutorado em Engenharia), Programa de Pós Graduação em Engenharia de Minas, Metalúrgica e de Materiais, Universidade Federal do Rio Grande do Sul, Porto Alegre. 2009.

3 Vasant JD, Kharde RR, Gujar UN. Fracture Split Connecting Rod for Automotive Engines. International Journal of Engineering Research and Development, 2012; 4: 37-41.

4 Carrara AR. Implantação de Sistemas BPMS Para a Gestão por Processo: uma análise crítica. Dissertação (Mestrado em Engenharia de Produção), Departamento de Engenharia de Produção, Escola Politécnica da Universidade de São Paulo, São Paulo. 2011.

5 Kelly LHF, Barros JGM. Análise da Implementação da Manutenção Produtiva Total". Anais III Simpósio de Excelência em Gestão e Tecnologia - SEGeT. Resende, 2006.

6 Neves SJA, Silva GL. Ajuste da Demanda da Produção em Empresa do Setor Automobilístico. Anais do VIII Simpósio de Excelência em Gestão e Tecnologia. Resende - RJ, 2011.

7 Favaretto F. Uma Contribuição ao Processo de Gestão da Produção Pelo Uso da Coleta Automática de Dados de Chão de Fábrica. Tese (Doutorado em Engenharia Mecânica), Programa de Pós-Graduação em Engenharia Mecânica da Escola de Engenharia de São Carlos (EESC). Universidade de São Paulo. São Carlos, 2001.

8 Rodero G, Freire JE, Santos EM, Faria MJ. Flexibilidade e Rapidez Voltadas ao Aumento da Competitividade de uma Micro Empresa do Setor Metalúrgico. Anais do XXX Encontro Nacional de Engenharia De Produção (ENEGEP). São Carlos-SP, 2010.

\footnotetext{
* Contribuição técnica ao $69^{\circ}$ Congresso Anual da ABM - Internacional e ao 14ํㅡㄹ ENEMET - Encontro Nacional de Estudantes de Engenharia Metalúrgica, de Materiais e de Minas, 21 a 25 de julho de 2014, São Paulo, SP, Brasil.
} 
9 Gonçalves JEL. As Empresas são Grandes Coleções de Processos. RAE. Revista de Administração de Empresas, v. 40, p. 6-19, São Paulo, 2000.

10 Neves JMS. A Implantação de Tecnologias da Informação como Fator de Competitividade nos Sistemas Produtivos e nos Negócios. Anais do XIII Simpósio de Engenharia de Produção (SIMPEP). Bauru, 2006.

11 Oliveira TH, Helleno AL. Sistema de Apoio à Gestão da Produção: indicadores de eficiência operacional- estudo de caso. Revista de Ciência \& Tecnologia, 2012; 17: 3952, São Paulo, 2012.

12 Peinado J, Graeml AR. Administração da produção: operações industriais e de serviços. Curitiba: UnicenP, 2007. 